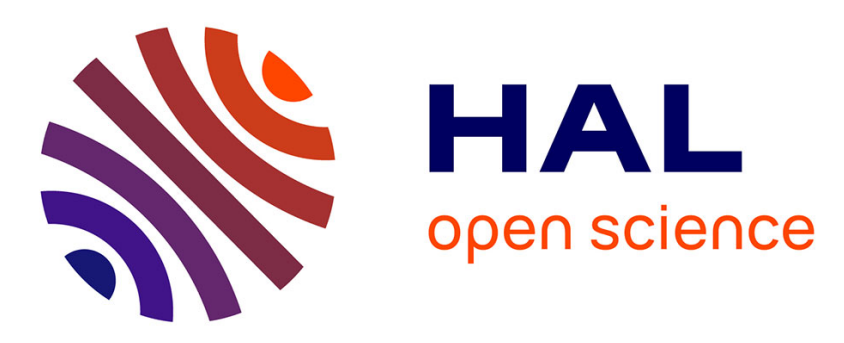

\title{
Classification of oyster habitats by combining wavelet-based texture features and polarimetric SAR descriptors
}

Olivier Regniers, Lionel Bombrun, Ioana Ilea, Virginie Lafon, Christian Germain

\section{To cite this version:}

Olivier Regniers, Lionel Bombrun, Ioana Ilea, Virginie Lafon, Christian Germain. Classification of oyster habitats by combining wavelet-based texture features and polarimetric SAR descriptors. IEEE International Geoscience and Remote Sensing Symposium 2015, 2015, Milan, Italy. pp.3890-3893. hal-01188201

\section{HAL Id: hal-01188201 \\ https://hal.science/hal-01188201}

Submitted on 28 Aug 2015

HAL is a multi-disciplinary open access archive for the deposit and dissemination of scientific research documents, whether they are published or not. The documents may come from teaching and research institutions in France or abroad, or from public or private research centers.
L'archive ouverte pluridisciplinaire HAL, est destinée au dépôt et à la diffusion de documents scientifiques de niveau recherche, publiés ou non, émanant des établissements d'enseignement et de recherche français ou étrangers, des laboratoires publics ou privés. 


\title{
CLASSIFICATION OF OYSTER HABITATS BY COMBINING WAVELET-BASED TEXTURE FEATURES AND POLARIMETRIC SAR DESCRIPTORS
}

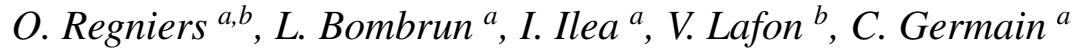 \\ ${ }^{a}$ Laboratoire IMS, Université de Bordeaux, Talence, France - ${ }^{\mathrm{b}}$ Géo-Transfert, Pessac, France
}

\begin{abstract}
In this study, we propose to evaluate the potential of combining very high resolution optical and SAR images for the classification of oyster habitats in tidal flats. To describe the classes of interest in both data, features are extracted by using wavelet-based texture features and polarimetric inter-band dependencies. A multisensor fusion scheme is then applied by adopting a maximum probability rule based on the outputs of SVM classifiers. Classification results show higher accuracies of detection of cultivated and abandoned oyster fields in comparison to classifications obtained using only texture features. This demonstrate the benefit of using both optical and SAR data for oyster habitats mapping in tidal flats.
\end{abstract}

Index Terms - texture, multi-sensor fusion, wavelet, SVM, classification, very high resolution, oyster habitats.

\section{INTRODUCTION}

Oyster farming is an important economic activity in France with several growing areas along the Atlantic coast. For the past decades, the oyster production in those areas has faced various sources of pressures related for instance to conflicts with other land uses, to a decreasing water quality as well as to the frequent bloom of toxic microplantonic species which forces the shell industry to sporadically cease sales. More recently, a worlwide spread virus caused frequent episodes of massive mortality of young oysters, leading to a significant reduction of the global oyster production. These ecological pressures contributes to a decrease of the economic viability of the oyster production resulting in the desaffection of numerous production site. These wild abandoned fields attract oyster spat which tend to grow rapidly becoming a nutrient sink and thereby a source of competition for cultivated oysters. In this constantly changing ecosystem, it becomes therefore necessary to develop new approaches designed to monitor this evolution by producing reliable cartography of cultivated and abandoned fields.

Some recent publications demonstrate the interest of the international community to detect and map oyster habitats in tidal flats using remote sensing data $[1,2,3]$. In these studies, the detection of oyster reefs is succesfully achieved by exploiting the radar backscattering properties specific to this land cover in SAR data (C- and X-band). In a previous study [4], we proposed another approach to detect cultivated oyster fields in very high resolution (VHR) panchromatic Pléiades data using texture analysis. This texture-based approach relies on the use of a multivariate model (Spherically Invariant Random Vector (SIRV) with a $\mathcal{G}^{0}$ distribution (SIRVg0 in the following) [5]) to represent the joint distribution of wavelet coefficients in each subband obtained by an orthogonal wavelet decomposition of the image. Parameters estimated during this modeling process were further used in a supervised classification procedure as a textural signature. Results obtained through this texture analysis enabled to efficiently detect oyster racks without significantly mistaking them with abandoned fields. Nonetheless, only the detection of cultivated fields was addressed in this former study.

In this paper, we propose to take a step further in the mapping of oyster fields by including abandoned fields in the classification process. Even though the spatial resolution of VHR SAR imagery is not sufficient to distinguish cultivated fields from wild abandoned fields, the specific backscattering properties of oysters in this type of data could help in the improvement of oyster detection. Therefore, in addition to texture features computed from optical data, descriptors derived from VHR SAR data are used and a multisensor fusion scheme is proposed to enhance the mapping accuracy. This fusion scheme is based on the probability outputs of SVM classifiers.

\section{STUDY SITE AND DATA}

The study site is located in the Arcachon Bay, a mesotidal lagoon of $180 \mathrm{~km}^{2}$ located in the South-West of France on the Atlantic coast. This area largely occupied by oyster farms is significantly affected by problems related to abandoned fields. In the Arcachon Bay, oysters are traditionally grown on racks of a few tens of meters to more than hundred meters long. These racks are set directly on the sediment in groups of two to five spaced by one to two meters (Fig. 1 line 1). When abandoned, oyster spat settle on the metal structure of the formerly exploited racks and the anisotropic aspect of the racks can most of the time still be observed in VHR remote sensing data (structured abandoned fields - Fig. 1 line 2). Besides, oysters can also be grown directly on the sediment without 


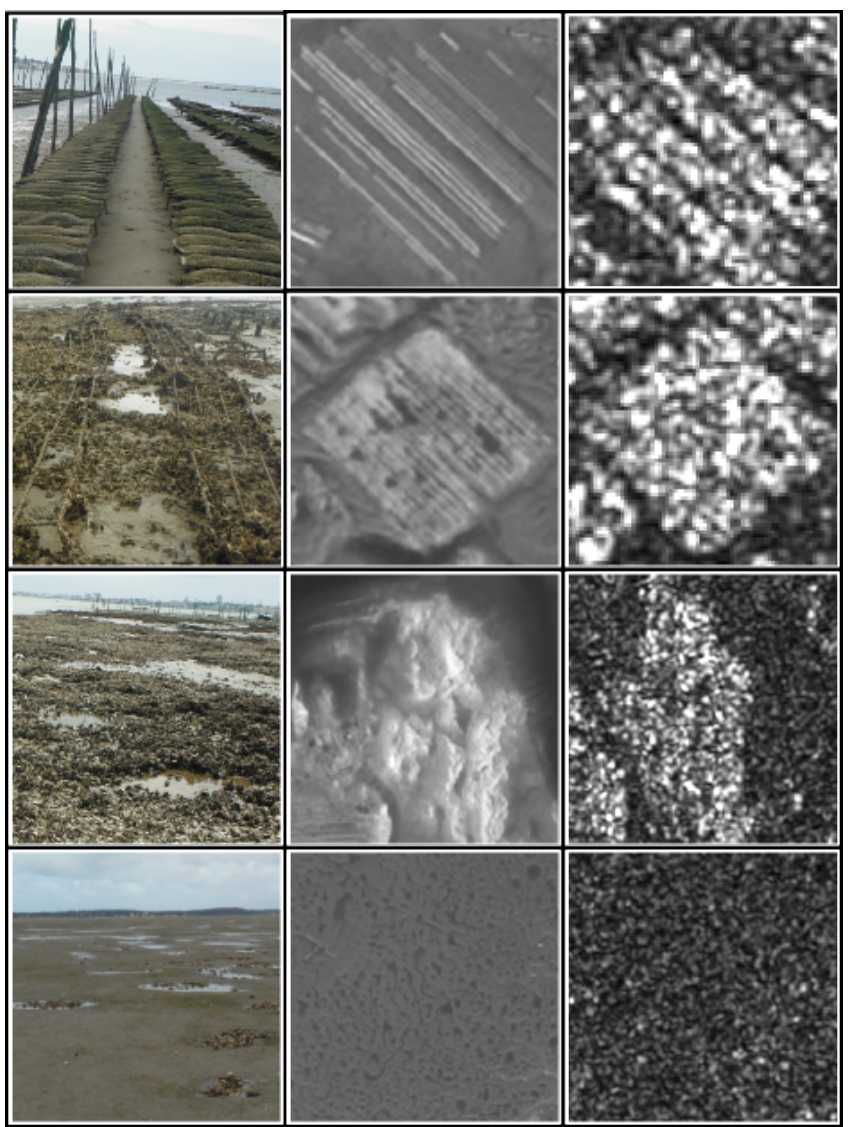

Fig. 1. Oyster fields typology. Left: field photography, center: extract of Pléiades panchromatic data, right: extract of TerraSAR-X image HH-polarization.

the use of racks to protect them. When abandoned, these wild growing areas are characterized by a more isotropic aspect (unstructured abandoned fields - Fig. 1 line 3). The rest of the land cover is mainly composed of clean areas and tidal flats either composed of sand or muddy sand (Fig. 1 line 4). Three classes are considered in the following for the classification process: cultivated fields, abandoned fields (either structured or unstructured) and tidal flats.

To perform the classification, we used two types of image data. The first is a panchromatic Pléiades image acquired at low tide in April 2013 with a spatial resolution of $0.5 \mathrm{~m}$ (PAN in the following). The second is a high resolution SpotLight TerraSAR-X data $(1 \mathrm{~m})$ with dual polarization $(\mathrm{HH} / \mathrm{VV})$ acquired in December 2009 at a higher tide level than the PAN image (TX in the following). It is noteworthy that the time gap between the two data acquisition dates as well as the difference in the tide level could be a source of misclassifications.

\section{METHODOLOGY}

The approach used in this study is divided in three steps. The first step is dedicated to the training phase during which features are extracted from a training database containing regions of interest (ROIs) representative of the different classes. In the next step, the supervised classification is carried out using a SVM classifier. Finally, in the last step, the classification results obtained with both PAN and TX images are fused and a final classification is produced.

\subsection{Feature Extraction}

Prior to the classification, a training database is created from both PAN and TX data by extracting ROIs representative of three classes: cultivated oyster racks, abandoned oyster fields and tidal flats (Fig. 1).

The ROIs extracted from the PAN data are further analyzed to extract texture features using a wavelet-based multivariate modeling approach as advocated in [4]. This approach works as follows. Previous to the feature extraction, a Stationary Wavelet Transform [6] with Daubechies filter db4 is applied on each ROI. Two scales and three orientations of decomposition are chosen leading to the production of six wavelet subbands. In each of these subbands, the wavelet coefficients located in a $3 \times 3$ neighborhood are clustered in a random vector $\mathbf{k}$. The distribution of these observations of the spatial dependency can be further modeled using a multivariate probability density function whose parameters are estimated according to the maximum likelihood principle. In [4], we previously demonstrated that a SIRV model $(\mathbf{k}=\sqrt{\tau} \mathbf{z})$ with an inverse gamma distributed multiplier $\tau$ [5] is a well suited model to characterize and detect oyster racks in panchromatic Pléiades image. This model is also used here. Hence, for each ROI, a textural feature vector containing an estimated covariance matrix and the parameters of the inverse gamma distributed multiplier is extracted for each wavelet subband.

In the case of the TX data, features derived from ROIs represent the intercanal dependency between $\mathrm{HH}$ and VV bands exprssed in $\mathrm{dB}$. Here, the observed vectors $\mathbf{k}$ contain for each pixel of an ROI the values in the HH and VV polarimetric bands. To model the distribution of the vectors $\mathbf{k}$, a bivariate Gaussian model is chosen. For each ROI, the extracted feature vector hence contains the parameters of the Gaussian distribution.

\subsection{Supervised Classification}

In order to avoid problems related to pixelwise classification (selection of the size of a sliding window, mixture of textures, high computational cost), the area to classify in the PAN data is first segmented using a Mean-Shift algorithm [7]. This presegmentation is deliberately over-segmented to preserve the outlines of small or narrow oyster fields. The parameters of 
the same multivariate models used during the training step are then estimated from each region defined through this segmentation. The same labeled mask representing the edges of the regions is used for both PAN and TX data even though it is initialy computed on the PAN image.

Once features were extracted from pre-segmented regions, a simillarity measure is calculated between the model estimated from each training ROI and the model estimated from each region. The chosen similarity measure is an approximation of a geodesic distance between two SIRVg0 models [8] for the PAN data and a Kullback-Leibler divergence between two multivariate Gaussians for the TX data. These similarities can be used in a SVM classifier by defining a Gaussian kernel $K$ such that:

$$
K\left(x, x^{\prime}\right)=\exp \left(-\frac{\operatorname{distance}\left(x, x^{\prime}\right)^{2}}{2 \sigma^{2}}\right)
$$

where $\left(x, x^{\prime}\right)$ is a couple of observations and $\sigma$ the standard deviation of the Gaussian kernel. The value of $\sigma$ is selected here to maximise the performance of the classifier.

The SVM classification is performed separately on the PAN and TX data. As the spatial resolution of the TX data does not allow to clearly distinguish between oyster racks and abandoned fields, these first two classifications only consider two classes, i.e. oyster fields (cultivated or abandoned) and tidal flats. For each region, an estimation of the class probabilities is computed for both PAN and TX data according to the approach proposed in [9]. These probability estimations are further used in the following fusion process.

\subsection{Fusion and Final Classification}

The fusion process is based on a absolute maximum probability rule and a threshold on the maximum. For each region to classify, only the maximum probability of both SVM classifiers is considered. If this maximum corresponds to the oyster fields class and if this maximum is inferior to a selected threshold, the corresponding region is classified as an oyster field. The other regions are put in an exclusion class containing those classifed as tidal flats and those rejected by the decision rule.

Finally, all the regions classified in the fusion process as oyster fields are once again classified using another SVM classifier based only on texture features derived from the PAN data. But, this time, all three classes (cultivated fields, abandoned fields and tidal flats) are included in the classification.

\section{RESULTS AND DISCUSSION}

An example of classification result on an area of interest in the Arcachon Bay is presented in Fig. 2-D. This result was obtained from the extracts of the PAN and TX data shown in Fig. 2-A and Fig. 2-B respectively. The difference in terms of tide level between both data is obvious with a few areas

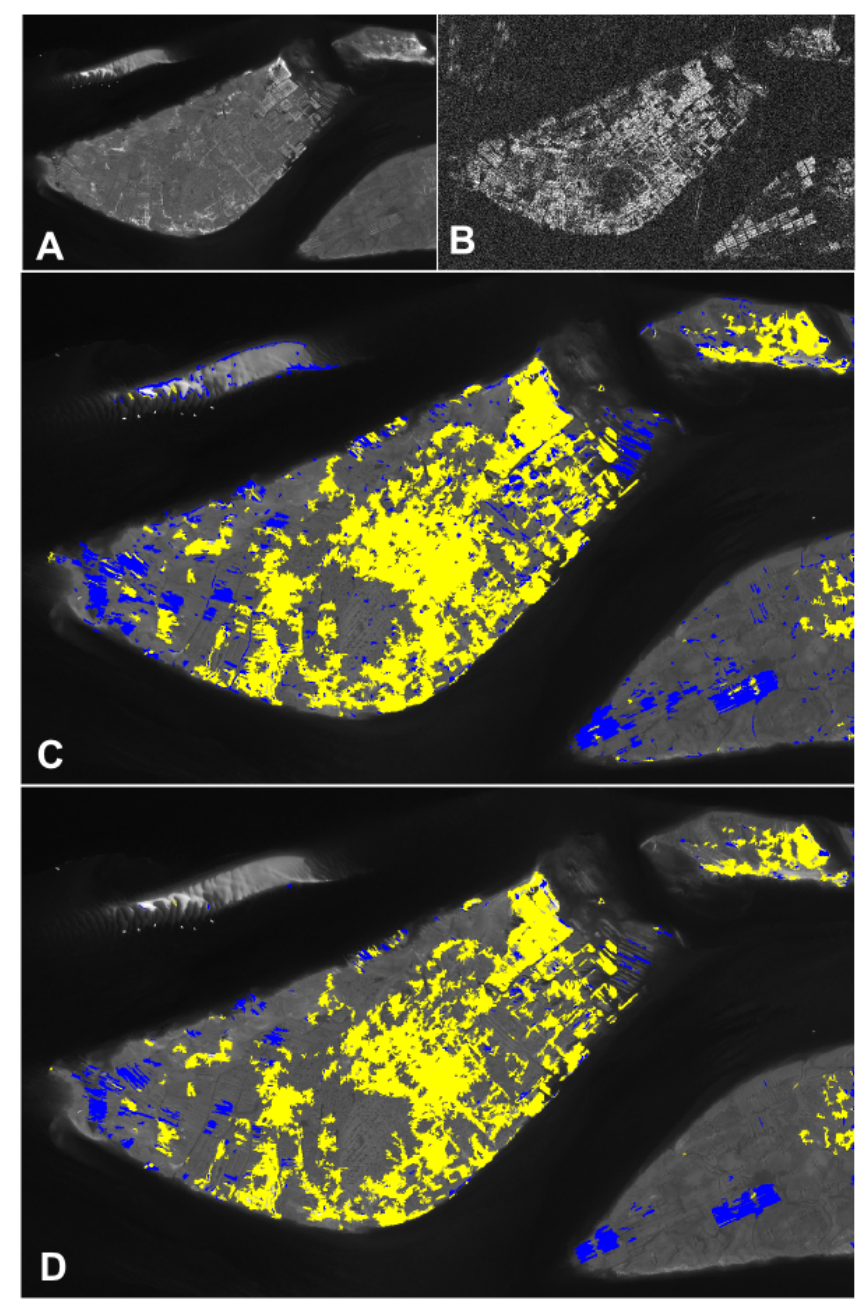

Fig. 2. (A) extract of PAN image, (B) extract of TX HH polarization, (C) SVM classification result using only the PAN image, (D) SVM classification result after fusion between PAN and TX. Cultivated oyster racks and abandoned fields are respectively represented in blue and yellow.

visible in the PAN image that are submerged in the TX data. Also, the time gap between the two images (December 2009 for the TX data and April 2013 for the PAN data) translates into occupational changes. This is for example the case in the southeastern part of the area where oyster racks are seen in the TX data and not in the PAN. To better illustrate the interest of the proposed fusion procedure, the classification result in Fig. 2-D is compared with the same result obtained with a SVM classifier and a probability threshold when using only texture features derived from the PAN data (Fig. 2-C).

In Fig. 2-C, most of the oyster fields (cultivated or abandoned) are well detected with only a few false negatives where oysters are partially submerged. These submerged areas are generally associated with a weaker contrast in the PAN data which does not allow to detect them properly when using tex- 
ture features. Besides, this classification result shows false positive detections with cultivated and abandoned fields significantly over-detected. This is particularly the case in small and narrow regions of only a hundred pixels defined during the pre-segmentation. These small regions might not contain a sufficient number of pixels to properly estimate the parameters of the SIRVg0 model and end up being misclassified. Also, most of these regions are defined in narrow areas where the textural content of the image is too poor to correspond to trained texture features. However, because of the narrowness of oyster racks, this over-segmentation of the PAN data is necessary to preserve an accurate detection of these structures.

On the other hand, in the fusion result (Fig. 2-D), most of these small over-detected areas are no longer misclassified and the contours of oyster racks and abandoned fields are more accurately detected. Even though the polarimetric TX data does not allow to differentiate between cultivated and abandoned oyster fields, the high backscattering coefficient associated with oyster fields in this data combined with texture features derived from the PAN data enables to detect oyster habitats with a high precision. The fusion based on the SVM maximum probability rule has proven to be adequate in this particular context of application. Furthermore, this decision rule prevents any misclassifications related to differences in terms of acquisition date or tide level between both data by giving a higher confidence to the PAN data when both classifications do not agree. Once identified during the fusion, the oyster fields can be accurately further classified as cultivated or abandoned by relying only on texture features as it was demonstrated earlier in [4].

\section{CONCLUSIONS}

We investigated the potential of combining VHR Pléiades panchromatic data with TerraSAR-X polarimetric data for the classification of oyster habitats in tidal flats. The proposed multi-sensor fusion scheme based on a maximum probability decision rule enabled to combine SVM classifications obtained with texture features and descriptors of the polarimetric channel dependencies. Results showed a higher qualitative accuracy of classification when fusing classifications derived from both sensors than when using only texture features derived from panchromatic data. In perspectives, this approach will be tested and validated on other pairs of Pléiades/TerraSAR-X data acquired on different oyster farming areas along the French Atlantic coast. These areas can indeed display a variety of oyster habitats which are not present in the Arcachon Bay, such as wild oysters growing on rocky beds.

\section{ACKNOWLEDGMENT}

This work was supported by the CNES (Centre National d'Études Spatiales) through the TOSCA project COQUILLE.
The authors also wish to thank CNES and its Thematic Users Commissioning team for providing the Pléiades data and the DLR for providing the TerraSAR-X data.

\section{REFERENCES}

[1] B. H. Choe, D. J. Kim, J. H. Hwang, Y. Oh, and W. M. Moon, "Detection of oyster habitat in tidal flats using multi-frequency polarimetric SAR data," Estuarine, Coastal and Shelf Science, vol. 97, pp. 28-37, 2012.

[2] M. Gade, S. Melchionna, K. Stelzer, and J. Kohlus, "Multi-frequency SAR data help improving the monitoring of intertidal flats on the German North Sea coast," Estuarine, Coastal and Shelf Science, vol. 140, pp. 32-42, 2014.

[3] J.-H. Ryu, J.-K. Choi, and Y.-K. Lee, "Potential of remote sensing in management of tidal flats: a case study of thematic mapping in the korean tidal flats," Ocean \& Coastal Management, vol. 102, pp. 458-470, 2014.

[4] O. Regniers, L. Bombrun, V. Lafon, A. Dehouck, C. Tinel, and C. Germain, "Wavelet based texture modeling for panchromatic very high resolution image classification: Application to oyster racks detection," in 2014 IEEE International Geoscience and Remote Sensing Symposium (IGARSS), 2014, pp. 5148-5151.

[5] C. C. Freitas, A. C. Frery, and A. H. Correia, "The polarimetric $\mathcal{G}$ distribution for SAR data analysis," Environmetrics, vol. 16, no. 1, pp. 13-31, 2005.

[6] M. Unser, "Texture classification and segmentation using wavelet frames," IEEE Transactions on Image Processing, vol. 4, no. 11, pp. 1549-1560, 1995.

[7] D. Comaniciu and P. Meer, "Mean shift: A robust approach toward feature space analysis," IEEE Transactions on Pattern Analysis and Machine Intelligence, vol. 24, no. 5, pp. 603-619, 2002.

[8] L. Bombrun, Y. Berthoumieu, N.-E. Lasmar, and G. Verdoolaege, "Multivariate texture retrieval using the geodesic distance between elliptically distributed random variables," in 2011 IEEE International Conference on Image Processing (ICIP), 2011, pp. 3637-3640.

[9] T.-F. Wu, C.-J. Lin, and R. C. Weng, "Probability estimates for multi-class classification by pairwise coupling," The Journal of Machine Learning Research, vol. 5, pp. 975-1005, 2004. 